\title{
Photoemission spectroscopy studies of the surface of GaN films grown by vapor phase epitaxy
}

\author{
Jian Ma, B. Garni, N. Perkins, W. L. O'Brien, ${ }^{\text {a) }}$ T. F. Kuech, and M. G. Lagally ${ }^{\text {b) }}$ \\ University of Wisconsin-Madison, Madison, Wisconsin 53706
}

(Received 12 July 1996; accepted for publication 16 September 1996)

\begin{abstract}
Surfaces of GaN films have been investigated with photoemission spectroscopy. The measured valence band is in good agreement with band structure calculations and correlates well with tunneling luminescence measurements performed on the same samples. The effect of $\mathrm{N}$ depletion on band structure is explored, clarifying disagreements in previous photoemission measurements. () 1996 American Institute of Physics. [S0003-6951(96)02448-5]
\end{abstract}

Because of its large direct band gap, gallium nitride $(\mathrm{GaN})$ has attracted interest for the development of bluegreen laser diodes and light emitting devices. ${ }^{1-3} \mathrm{GaN}$ films can be synthesized either in the hexagonal wurtzite structure $(\alpha-\mathrm{GaN})$ or in the cubic zinc-blende phase $(\beta-\mathrm{GaN}),{ }^{4,5}$ although $\beta$-GaN films are usually very difficult to grow. Because of the difficulty in making structurally and compositionally high quality films, the electronic structure of $\mathrm{GaN}$ is far less well known than that of some other Ga-V semiconductors, e.g., GaP, GaAs, and GaSb. Numerous band structure calculations have been performed. ${ }^{6-11}$ They have been compared with optical measurements ${ }^{12}$ and limited photoemission spectroscopy measurements. ${ }^{13-19}$ There have as yet been no systematic measurements on surfaces of $\mathrm{GaN}$ that are believed to be clean. Different groups do not even agree on the binding energy of $\mathrm{Ga}(3 d)$ core levels. ${ }^{13-19}$ In this Letter, we report a study of the surface of GaN films grown by halide vapor phase epitaxy (HVPE) using synchrotron radiation angle-integrated photoemission spectroscopy. Our measurements indicate that the variability in results obtained by different groups is related to contaminants on and the stoichiometry of the surface of $\mathrm{GaN}$.

We used $n$-type (0001)-oriented single-crystal GaN films with the wurtzite structure, grown on (0001) sapphire by halide vapor phase epitaxy. The film growth procedures have been described elsewhere. ${ }^{20}$ The samples exhibit a low reactivity to surface contamination or chemical modification. They were transferred into the photoemission chamber at the Synchrotron Radiation Center (SRC) through a load-lock without being baked. The photoemission chamber has a base pressure of $1.4 \times 10^{-10}$ Torr and is equipped with an ion sputtering gun and a station for heating the sample through electron beam bombardment. The energy resolution of our measurements, determined by the measurement of the Fermi edge of a sputter-cleaned copper foil, is $0.2 \mathrm{eV}$. The angle of incidence of the light is $45^{\circ}$.

Figure 1 shows photoemission energy distribution curves using a photon energy of $50 \mathrm{eV}$ taken on the surface of a $\sim 22 \mu \mathrm{m}$ thick GaN film treated in different manners. Spectrum (a) was obtained on an as-grown surface without any post-growth treatment. The main features are the valence

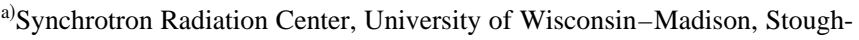
ton, WI 53589.

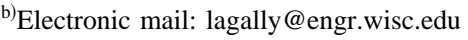

band between $2.7 \mathrm{eV}$ and $13.6 \mathrm{eV}$, and the $\mathrm{Ga}(3 d)$ core level at $22.2 \mathrm{eV}$. The intensity of $\mathrm{Ga}(3 d)$ line is relatively weak compared with the intensity from the valence band. Two peaks at $18.3 \mathrm{eV}$ and $15.6 \mathrm{eV}$ are assigned to surface contaminants for reasons stated later. After we exposed the asgrown surface to the zero-order light from synchrotron radiation for about $20 \mathrm{~min}$, which is equivalent to a photodesorption cleaning procedure, the peak at $18.3 \mathrm{eV}$ and the shoulder at $15.6 \mathrm{eV}$ were reduced significantly, as shown by spectrum (b) in Fig. 1, while the main valence peak and $\mathrm{Ga}(3 d)$ line shifted towards smaller binding energy. These changes are consistent with a modification in the local electronic states upon removal of a thin overlayer of contaminants absorbed on the clean GaN surface. The changes in the surface charge cause a change in the local electrostatic potential and therefore a rigid shift in energy of the electronic states with respect to the Fermi level. ${ }^{21}$ The photoelectron emission in the gap observed in spectra (a) and (b) of Fig. 1

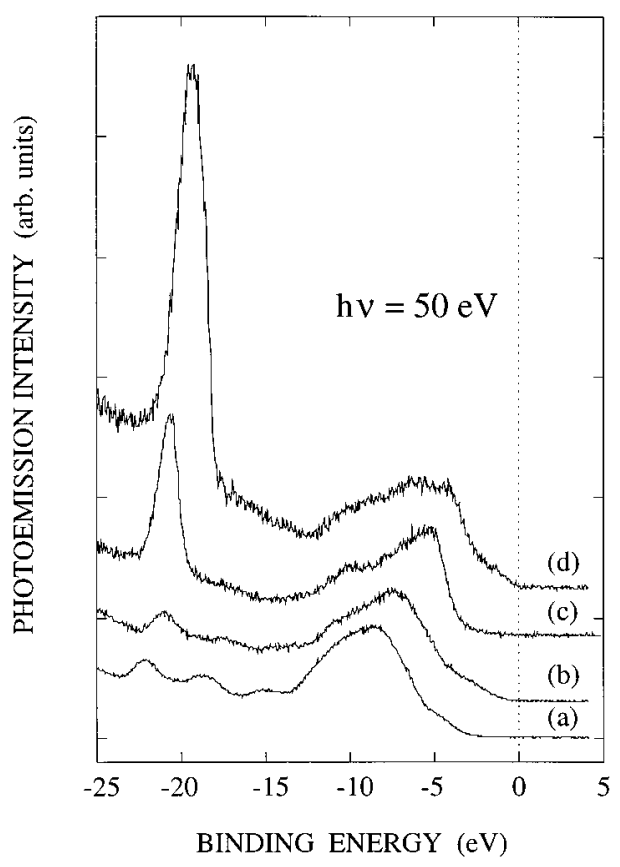

FIG. 1. Photoemission spectra for the valence band and $\mathrm{Ga}(3 d)$ core level taken on the surfaces of GaN treated in different ways: (a) as-grown; (b) cleaned with exposure to the zero-order light from synchrotron radiation; (c) cleaned by electron beam heating for the bulk; and (d) cleaned by $\mathrm{Ar}^{+}$ sputtering. 


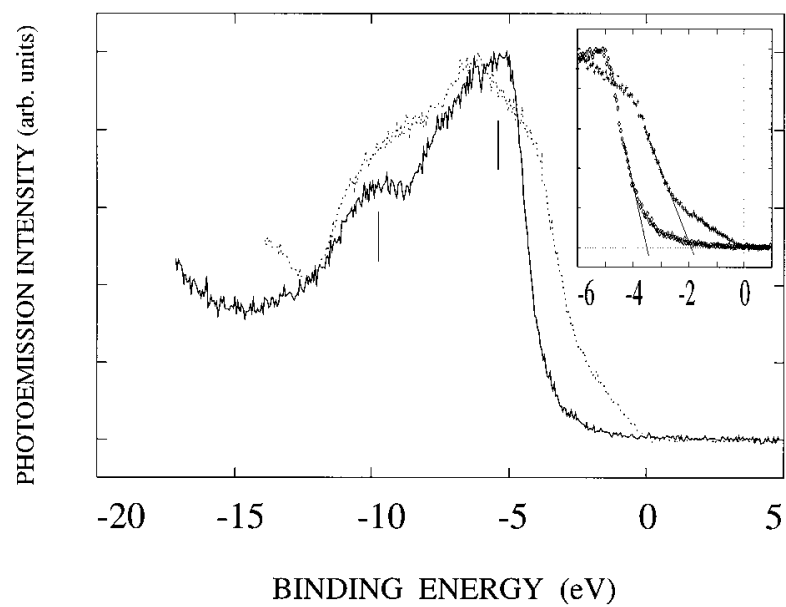

FIG. 2. Valence band photoemission spectra of clean surface of GaN prepared by electron beam heating (solid curve) and (b) $\mathrm{Ar}^{+}$sputtering (dotted curve). The inset shows the blow-up of the spectra near the valence-band edge.

also seems to be related to contaminants. They are not likely Ga-related since they can be removed by thermal heating as shown in spectrum (c).

Spectrum (c) in Fig. 1 was obtained for the surface of $\mathrm{GaN}$ subjected to electron bombardment on the back of the sample. The sample was heated at about $500-600{ }^{\circ} \mathrm{C}$ several times for several minutes. It was then flashed to about $900{ }^{\circ} \mathrm{C}$ for $2 \mathrm{~s}$. After such a procedure, the peaks at $18.3 \mathrm{eV}$ and $15.6 \mathrm{eV}$ disappear. The intensity of the $\mathrm{Ga}(3 d)$ line increases dramatically. The main features in the valence band become clearly resolved. We believe that the procedure used to obtain spectrum (c) gives a reasonably clean surface.

Curve (d) in Fig. 1 was obtained on a surface that was sputter-etched with $\mathrm{Ar}^{+}$for about $30 \mathrm{~min}$. It has been reported ${ }^{18}$ that this procedure will change the surface stoichiometry of GaN significantly because of loss of $\mathrm{N}$ in the surface layers. As one can see, the $\mathrm{Ga}(3 d)$ peak intensity increases at least a factor of 2 . At the same time, its position changes from $20.7 \mathrm{eV}$ to $19.4 \mathrm{eV}$, shifting towards smaller binding energy.

Figure 2 shows a closer look at the valence band for the clean near-stoichiometric GaN surface (solid line) and its comparison with a clean non-stoichiometric surface (dotted line). For the near-stoichiometric surface, the dominant structures are centered at $5.4 \mathrm{eV}$ and $9.7 \mathrm{eV}$ below $E_{f}$. According to the band structure calculations, the $5.4 \mathrm{eV}$ feature is mainly nitrogen $p$-derived, and the $9.7 \mathrm{eV}$ one is associated with hybridized $\mathrm{Ga}(s)$ and $\mathrm{N}(p)$ states. The binding energies of these two features are $1.9 \mathrm{eV}$ and $6.2 \mathrm{eV}$ if one uses the valence band maximum (VBM) determined using a linear extrapolation as shown in the inset of Fig. 2 as energy reference. The location of these two maxima in the photoemission spectrum appears to fit very well with the major density of states (DOS) features predicted in band structure calculations. ${ }^{10}$ In Fig. 2, the VBM is $3.5 \pm 0.1 \mathrm{eV}$ below the experimental Fermi level, which can be viewed as the band gap value if we assume that the Fermi level is right at the conduction band minimum (CBM), consistent with our separate photoluminescence measurement ${ }^{22}$ which shows a 3.37

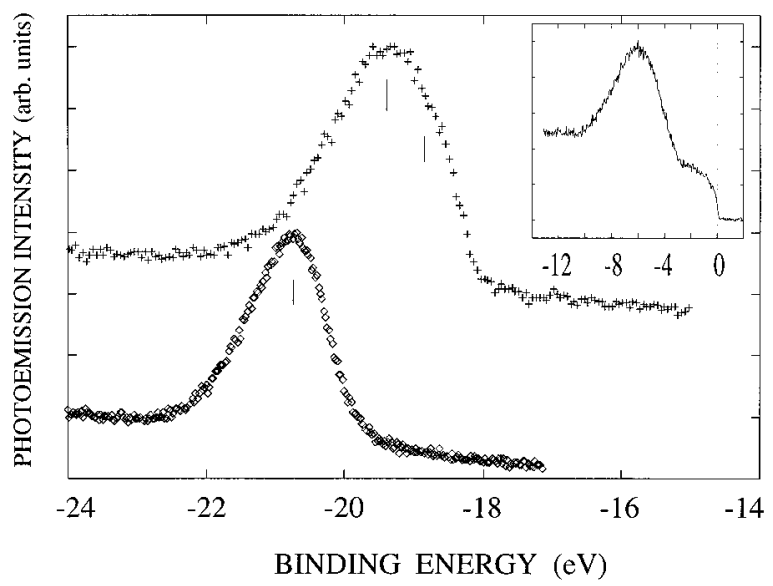

FIG. 3. Photoemission spectra of the $\mathrm{Ga}(3 d)$ core level of (diamonds) clean near-stoichiometric and (crosses) N-depleted surfaces of GaN. The inset shows the metallization of the surface upon excessive $\mathrm{Ar}^{+}$sputtering.

$\mathrm{eV}$ band gap for these films. The Fermi energy is determined from a sputter-cleaned copper foil in good electrical contact with the sample. The depletion of $\mathrm{N}$ in the surface changes the spectrum significantly: the lineshape of the valence band changes and states appear in the gap. While the feature associated with hybridized $\mathrm{Ga}(s)$ and $\mathrm{N}(p)$ states remains intact, the nitrogen $p$-derived structure has been reduced in intensity.

We have repeated these measurements for photon energies between $33 \mathrm{eV}$ and $150 \mathrm{eV}$. Except for a change of relative intensity for the different features because the photoelectron cross sections vary with energy, no detectable dispersion was observed with photon energy, confirming that the observed features are associated with the structure of the initial density of states. ${ }^{18}$

We have reported elsewhere ${ }^{20}$ measurements on samples grown in the same manner using scanning tunneling microscopy (STM) and scanning tunneling luminescence (STL). In the tunneling luminescence measurements, holes are injected into the sample by applying a bias voltage between the sample and STM tip. The injected holes thermalize rapidly to the top of the valence band and recombine with the electrons at the bottom of the conduction band and in defect states in the gap to produce luminescence. The intensity of the luminescence is proportional to the tunneling current, which is closely related to the density of states for the GaN valence band. The lineshape of the valence band for the nearstoichiometric surface (Fig. 2) correlates very well with our luminescence measurements. ${ }^{20}$ Both the valence band photoemission intensity and the luminescence intensity peak at about $5.0 \mathrm{eV}$ with respect to the Fermi energy.

Figure 3 shows spectra of the $\mathrm{Ga}(3 d)$ core level of the e-beam-cleaned and the $\mathrm{Ar}^{+}$-sputtered surfaces taken at a photon energy of $50 \mathrm{eV}$. There is no indication of the $\mathrm{N}(2 s)$ peak because the cross section of $\mathrm{N}(2 s)$ state is very low at this photon energy. Using a photon energy of $150 \mathrm{eV}$ we have, however, seen the state appearing as a small bump centered at $17.5 \mathrm{eV}$ on the lower-binding-energy side of the $\mathrm{Ga}(3 d)$ peak. The binding energy of $\mathrm{Ga}(3 d)$ is determined to be $20.7 \pm 0.1 \mathrm{eV}$, in excellent agreement with $20.6 \pm 0.2 \mathrm{eV}$ reported by Bermudez et al. ${ }^{19}$ who prepared clean surfaces 
of GaN using a somewhat different approach, but is somewhat larger than the $19.7 \mathrm{eV}$ values reported earlier. ${ }^{13} \mathrm{We}$ found no indication of charging typically associated with photoelectron emission from insulators, as expected since our samples conduct well. Such charging would shift spectra to higher binding energy. The $\mathrm{Ga}(3 d)$ core level spectrum from a non-stoichiometric surface, resulting from $30 \mathrm{~min}$ $\mathrm{Ar}^{+}$sputtering (Fig. 3, crosses), shows an indication of two peaks, centered at $19.4 \mathrm{eV}$ and $18.8 \mathrm{eV}$. The peak at $19.4 \mathrm{eV}$ agrees with $19.7 \pm 0.1 \mathrm{eV},{ }^{13,16}$ which we believe is associated with strongly nitrogen-depleted $\mathrm{GaN}$ given the way the surface is prepared. The second peak at $18.7 \mathrm{eV}$ is very close to the binding energy of $\mathrm{Ga}(3 d)$ in a $\mathrm{Ga}$ metal overlayer (18.4 $\mathrm{eV}),{ }^{23}$ indicating the possible formation of Ga metal clusters on the surface. However, the number of clusters is not large enough to make the surface metallic as we did not see photoemission intensity at $E_{f}$. We did see the emission at $E_{f}$ after further $\mathrm{Ar}^{+}$sputtering for about $15 \mathrm{~min}$ as shown in the inset of Fig. 3, indicating the formation of a metallic phase on the surface.

In summary, we have performed photoemission spectroscopy measurements on thick GaN films grown by halide vapor phase epitaxy on sapphire. The valence band spectrum obtained on the clean surface is in good agreement with band structure calculations, and correlates very well with tunneling luminescence measurements performed on the same samples. ${ }^{20}$ Our results of $\mathrm{Ar}^{+}$sputtered surfaces show that the discrepancy in binding energy of $\mathrm{Ga}(3 d)$ reported by different groups can be attributed to nitrogen depletion in the surface.

One of the authors (J.M.) would like to acknowledge helpful discussions with Dr. M. Onellion. The assistance from the staff of SRC, particularly M. Bissen and D. Wallace, is gratefully acknowledged. This work was supported by ONR, Electronics Division and by NSF Grant No.
DMR91-21074, the Naval Research Laboratory, and the ARPA URI on Visible Light Emitters. SRC is supported by NSF Grant No. DMR95-31009.

${ }^{1}$ R. F. Davis, Proc. IEEE 79, 702 (1991); Physica B 185, 1 (1993), and references therein.

${ }^{2}$ J. I. Pankove, Mater. Res. Soc. Symp. Proc. 97, 409 (1987).

${ }^{3}$ I. Akasaki, H. Amano, N. Koide, M. Kotaki, and K. Manabe, Physica B 185, 428 (1993).

${ }^{4}$ O. Brandt, H. Yang, B. Jenichen, Y. Suzuki, L. Däweritz, and K. H. Ploog, Phys. Rev. B 52, R2253 (1995).

${ }^{5}$ D. E. Lacklison, J. W. Orton, I. Harrison, T. S. Cheng, L. C. Jenkins, C. T. Foxon, and S. E. Hooper, J. Appl. Phys. 78, 1838 (1995).

${ }^{6}$ S. Bloom, G. Harbeke, E. Meier, and I. B. Ortenburger, Phys. Status Solidi B 66, 161 (1974).

${ }^{7}$ S. J. Bloom, J. Phys. Chem. Solids 32, 2027 (1971).

${ }^{8}$ D. Jones and A. H. Lettington, Solid State Commun. 11, 701 (1972).

${ }^{9}$ J. Bourne and R. L. Jacobs, J. Phys. C 5, 3462 (1972).

${ }^{10}$ M.-Z. Huang and W. Y. Ching, J. Phys. Chem. Solids 46, 977 (1985).

${ }^{11}$ B. J. Min, C. T. Chan, and K. M. Ho, Phys. Rev. B 45, 1159 (1992).

${ }^{12}$ C. G. Olson and D. W. Lynch, Phys. Rev. B 24, 4629 (1981), and references therein.

${ }^{13}$ J. Hedman and N. Mårtensson, Phys. Scr. 22, 176 (1980).

${ }^{14}$ R. Carin, J. P. Deville, and J. Werckmann, Surf. Interface Anal. 16, 65 (1990).

${ }^{15}$ D. Troost, H. U. Baier, A. Berger, and W. Mönch, Surf. Sci. 242, 324 (1991).

${ }^{16}$ L. A. Delouise, J. Vac. Sci. Technol. A 10, 1637 (1992).

${ }^{17}$ X.-Y. Zhu, T. Huett, M. Wolf, and J. M. White, Appl. Phys. Lett. 61, 3175 (1992).

${ }^{18}$ R. W. Hunt, L. Vanzetti, T. Castro, K. M. Chen, L. Sorba, P. I. Cohen, W. Gladfelter, J. M. Van Hove, J. N. Kuznia, M. Asif Khan, and A. Franciosi, Physica B 185, 415 (1993).

${ }^{19}$ V. M. Bermudez, R. Kaplan, M. A. Khan, and J. N. Kuznia, Phys. Rev. B 48, 2436 (1993).

${ }^{20}$ B. Garni, Jian Ma, N. Perkins, Jutong Liu, T. F. Keuch, and M. G. Lagally, Appl. Phys. Lett. 68, 1380 (1996).

${ }^{21} \mathrm{G}$. Margaritondo and J. H. Weaver, in Methods of Experimental Physics: Surfaces, edited by M. G. Lagally and R. L. Park (Academic, New York, 1985).

${ }^{22} \mathrm{~J}$. Liu and T. F. Kuech (unpublished).

${ }^{23}$ E. Antonides, E. C. Janes, and G. A. Sawatzky, Phys. Rev. B 15, 1669 (1977). 